\title{
Bacteraemia due to Campylobacter fetus subsp. fetus in a liver transplanted patient
}

\author{
Tito Del Gaudio', Maria Antonietta Distasi', Mariella Porzio', Antonio Parisi², Michele Cannone ${ }^{3}$, \\ Nicola Minervino ${ }^{3}$, Adriana Mosca ${ }^{4}$, Giuseppe Miragliotta ${ }^{4}$ \\ I Laboratorio di Analisi P.O. Andria AUSL BAT, \\ 2 Istituto Zooprofilattico Sperimentale Putignano, \\ 3 U.O. Medicina P.O. Conosa AUSL BAT, \\ 4 Sez. di Microbiologia, Dip. MIDIM, Università degli Studi di Bari.
}

Key words: Campylobacter fetus, bacteraemia.

Batteriemia da Campylobacter fetus subsp. fetus in un paziente con trapianto epatico

\section{SUMMARY}

Campylobacter fetus is a mobile, curved or spiral-shaped Gram negative bacillus. We report a case of bacteraemia due to C. fetus subsp. fetus isolated from a 47 -year-old male patient liver transplanted, with underlying cardiovascular disease and receiving immunosuppressive therapy with cyclosporine. This patient was admitted to the hospital with fever and pain to both the legs.After admission, the microorganism isolated from blood-culture was identified as Campylobacter fetus subsp fetus either by traditional methods or by I6S rRNA gene sequencing. Our observation emphasizes the possibility that such unusual microorganisms can be isolated from immunosuppressed patients and, therefore, particular attention should be paid to the strategies for their detection.

\section{INTRODUZIONE}

Campylobacter fetus è un batterio Gram negativo spiraliforme, mobile per la presenza di un flagello polare.

Patogeno per ovini e bovini, nell’uomo può essere responsabile, soprattutto in caso di immunosoppressione, di infezione sistemica caratterizzata da batteriemia cui possono far seguito endocardite, salpingite ed ascessi polmonari. C. fetus è stato associato a casi di aborto e colonizzazione del liquido amniotico. In tale microrganismo è stato evidenziato uno strato proteico (proteina S) di elevato peso molecolare che, legato al lipopolisaccaride mediante la porzione $\mathrm{N}$-terminale è in grado di conferire resistenza all'azione del complemento ed alla fagocitosi neutrofila. Si possono distinguere due sottospecie di $C$. fetus: $C$. fetus subsp. venerealis e $C$. fetus subsp. fetus, in grado di invadere l'ospite con meccanismi patogenetici diversi. Segnaliamo un caso di batteriemia da $C$. fetus subsp. fetus isolato da un paziente sottoposto a trapianto epatico.

\section{CASO CLINICO}

Paziente M.N., 47 anni, già epatotrapiantato per cirrosi epatica HCV-correlata, giunto all'osservazione del P.O. di Canosa di Puglia per la comparsa di dolori diffusi agli arti inferiori, febbricola $\left(37.3^{\circ} \mathrm{C}\right)$ e lieve leucocitosi neutrofila. $\mathrm{E}$ in trattamento, all'atto del ricovero, con corticosteroidi (Deltacortene $5 \mathrm{mg}$ : $2 \mathrm{cp} / \mathrm{die}$ ) e ciclosporina (Sandimmun Neoral $100 \mathrm{mg} / \mathrm{die}$ ). Gli esami ematochimici confermano un incremento della percentuale dei leucociti neutrofili ed un modico aumento di VES e CRP. Rx torace e Rx diretta addome non evidenziano focolai infettivo-infiammatori in corso. Durante la degenza, la TC raggiunge i $39.7^{\circ} \mathrm{C}$ : viene eseguita l'emocoltura (presso l’U.O. di Patologia clinica del P.O. di Andria), viene sospesa la terapia con ciclosporina per 24 ore ed inizia la terapia antibiotica con levofloxacina (Tavanic 500 $\mathrm{mg} /$ die). Il paziente viene dimesso apiretico, con ripristino della terapia immunosoppressiva.

\section{MATERIALI E METODI}

L'emocoltura è stata eseguita utilizzando il sistema BacT ALERT FA bioMérieux. Il sistema ha segnalato la positività del campione dopo 6 giorni di incubazione. L’osservazione microscopica previa colorazione di Gram e blu di metilene, ha rivelato la presenza di microrganismi Gram negativi di aspetto spirillare (Figura I). Successivamente alla semina su piastre convenzionali e su agar Blaser Wang, l'emocoltura è stata incubata a $42{ }^{\circ} \mathrm{C}$ in atmosfera di $\mathrm{CO}_{2}$ utilizzando il generatore GasPak EZ Campy Container System (Becton Dickinson). L'identificazione è stata eseguita mediante sequenziamento del gene 16S rRNA con sistema Big Dye Terminator Cycle Sequencing v3.1 Ready Reaction Kit (Applied Biosystems). L'antibiogramma è stato eseguito con il metodo Kirby-Bauer su Muller Hinton agar addizionato con sangue di montone. L'incubazione è avvenuta nelle stesse condizioni della coltura primaria.

\section{RISULTATI}

Le colonie, sviluppatesi dopo $48 \mathrm{~h}$ di incubazione, sono state identificate come appartenenti alla specie $C$. fetus subsp. fetus. L'antibiogramma ha rivelato la sensibilità di tale ceppo di C. fetus subsp. fetus ad amikacina, gentamicina, claritromicina, eritromicina, clindamicina, ofloxacina, ciprofloxacina, ceftriaxone; vi era resistenza a ceftazidime ed imipenem.

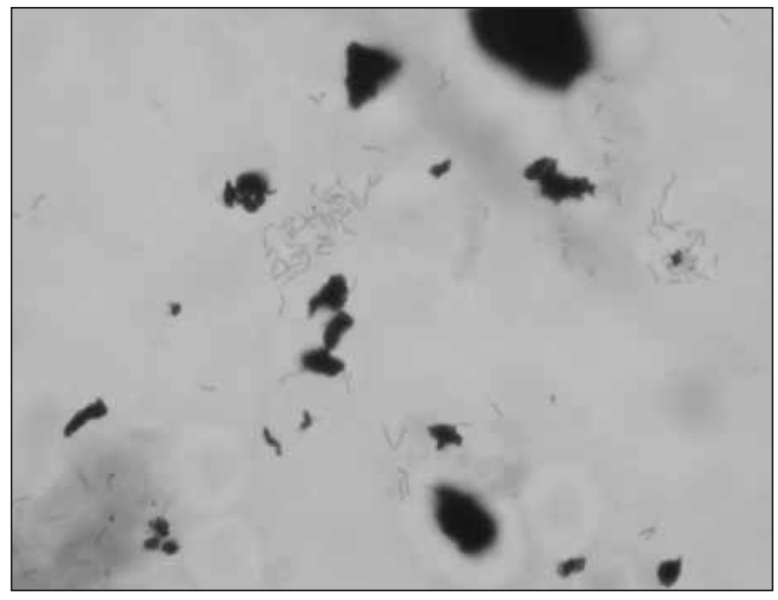

Figura I. Osservazione microscopica dopo colorazione di Gram. Presenza di bacilli Gram negativi dall'aspetto spirillare.

\section{CONCLUSIONI}

La nostra osservazione sottolinea la necessità di non trascurare, nei pazienti immunocompromessi, la possibilità di 
riscontrare microrganismi insoliti che necessitano di particolari accorgimenti sia per quanto riguarda l'isolamento che l'identificazione. Appare indispensabile, nei casi di positività dell'emocoltura, l'allestimento di preparati per l'osservazione microscopica diretta che permettano di orientare in maniera ottimale l'esecuzione delle subculture. È inoltre di fondamentale importanza, in casi come quelli descritti, il prolungamento dei tempi di incubazione dell'emocoltura. Risulta inoltre di particolare interesse, nel caso descritto, la presenza di resistenza ad imipenem, antibiotico di prima scelta nelle infezioni gravi.

\section{BIBLIOGRAFIA}

1. Dumoulard B, Wallet F, Glowacki F, Courcol RJ. Campylobacter fetus subsp fetus bacteremia in a female patient infected by the hepatitis $C$ virus. Ann Biol Clin. (Paris). 2004; 62(5): 587-9.

2. Epifane G, Barbon S, Sadino G, Bouchet D, Suárez ME, Mangiaterra S.
Bacteremia due to Campylobacter fetus isolated by conventional methods from an immunocompromised patient. Rev Argent Microbiol. 2007;39(1): 34-7.

3. Gazaigne L, Legrand P, Renaud B, et al. Campylobacter fetus bloodstream infection: risk factors and clinical features. Eur J Clin Microbiol Infect Dis. 2008; 27(3): 185-9.

4. Heng AE, De Champs C, Souweine B, Guy L, Sirot J, Deteix P. Campylobacter fetus bacteraemia in a renal graft recipient. Nephrol Dial Transplant 2002; 17: 689-90.

5. Krause R, Ramschak-Schwarzer S, Gorkiewicz G, et al. Recurrent septicemia due to Campylobacter fetus and Campylobacter lari in an immunocompetent patient. Infection. 2002; 30(3): 171-4.

6. Linton D, Owen RJ, Stanley J. Rapid identification by PCR of the genus Campylobacter and five Campylobacter species enteropathogenic for man and animals. Research in Microbiology. 1996; 147: 707-18.

7. Monno R, Rendina M, Ceci G, et al. Campylobacter fetus bacteremia in an immunocompromised patient: case report and review of the literature. New Microbiol. 2004; 27(3): 281-5.

8. Viejo G, Gomez B, De Miguel D, Del Valle A, Otero L, De La Iglesia P. Campylobacter fetus subspecies fetus bacteremia associated with chorioamnionitis and intact fetal membranes. Scand J Infect Dis. 2001; 33(2): 126-7. 